\title{
Molecular Dynamics of Carbon Nanotubes Deposited on a Silicon Surface via Collision: Temperature Dependence
}

\author{
Leton C. Saha, Shabeer A. Mian, Hyojeong Kim, Joyanta K. Saha, Mohammad A. Matin, and Joonkyung Jang* \\ Department of Nanomaterials Engineering, Pusan National University, Miryang 627-706, Korea \\ ${ }^{*}$ E-mail: jkjang@pusan.ac.kr \\ Received October 28, 2010, Accepted December 1, 2010
}

\begin{abstract}
We investigated how temperature influences the structural and energetic dynamics of carbon nanotubes (CNTs) undergoing a high-speed impact with a Si (110) surface. By performing molecular dynamics simulations in the temperature range of $100-300 \mathrm{~K}$, we found that a low temperature CNT ends up with a higher vibrational energy after collision than a high temperature CNT. The vibrational temperature of CNT increases by increasing the surface temperature. Overall, the structural and energy relaxation of low temperature CNTs are faster than those of high temperature CNTs.
\end{abstract}

Key Words: Carbon nanotube, Collision, Molecular dynamics, Surface deposition, Relaxation

\section{Introduction}

Reinforcement with carbon nanotubes (CNTs) has been widely used in synthesizing composite materials. ${ }^{1-7}$ Such composite materials are often deposited on surfaces by means of spraying ${ }^{8,9}$ at high velocity (more than several hundred $\mathrm{km} / \mathrm{s}$ ). Cold spraying ${ }^{10,11}$ is one of the techniques that is used to prepare CNT based composite materials. ${ }^{12,13}$ Before spraying, the CNT composites are heat-treated at high temperature (3000 $46000 \mathrm{~K}^{8}$ or $400-900 \mathrm{~K}^{12,13}$ ). CNTs suffer from deformation caused by a high velocity impact with surface and heat treatment. It would be interesting to study the dynamics and molecular details for the deformation and energy excitation of CNT in such process. There have been several simulation studies on how temperature affects the structure ${ }^{14,15}$ or conductivity ${ }^{16,17}$ of a single isolated CNT. We are also aware of simulation studies on the structural stability of CNT colliding with a surface, ${ }^{18,19,20}$ but the effect of temperature was not taken into account. Herein, we study how temperature affects the CNT deposition under a high velocity impact with a silicon surface. By varying temperatures of both the CNT and the surface, we dynamically investigate the deformation and energy excitation of the CNT. Our molecular dynamics (MD) simulation shows that the vibrational temperature of CNT after collision increases with raising the surface temperature. A CNT initially equilibrated at low temperature turns out more efficient in relaxing its deformed structure and excited vibrational energy. Interestingly, a low temperature CNT ends up with a higher vibrational temperature after collision than a CNT with a high initial temperature. We ascribe this heat transfer from the surface at high temperature to the CNT at low temperature.

\section{Simulation Details}

A $(6,6)$ single-walled CNT impacting on the Si (110) surface is shown in Figure 1a. The CNT, made from $384 \mathrm{C}$ atoms, was $3.8 \mathrm{~nm}$ long and $0.81 \mathrm{~nm}$ in diameter. The CNT was shown to be delivered onto the surface with a projectile speed, $v_{p}$, where the arrow in Figure 1a denotes its direction. $v_{p}$ was chosen to be $5 \mathrm{~km} / \mathrm{s}$ which is more than three times higher than that in typical cold spray processes. ${ }^{12,13}$ In Figure 1a, the temperature of CNT, $\mathrm{T}_{\mathrm{CNT}}$, and the surface temperature, $\mathrm{T}_{\mathrm{Si}}$, are 100 and $300 \mathrm{~K}$, respectively. The $\mathrm{Si}(110)$ surface consisted of a slab of Si crystal with a thickness of $3.43 \mathrm{~nm}$. The atoms in the bottom layer of the slab were fixed throughout the entire simulation. The surface has lateral dimensions of $15.61 \mathrm{~nm} \times 8.97 \mathrm{~nm}$. The periodic boundary conditions ${ }^{21}$ were imposed to emulate a laterally infinite surface. The length of simulation box along the $Z$ direction was taken to be $50 \mathrm{~nm}$, in order to avoid the periodicity in the Z-direction. The total number of Si atoms was 24360 for the surface. The CNT and surface were equilibrated separately (to give desired $\mathrm{T}_{\mathrm{CNT}}$ and $\mathrm{T}_{\mathrm{Si}}$ ) by running constant temperature (NVT) $\mathrm{MD}^{21}$ simulations. $\mathrm{T}_{\mathrm{CNT}}$ values of 100 and $300 \mathrm{~K}$ and $\mathrm{T}_{\mathrm{Si}}$ values of $100,150,200,250$ and $300 \mathrm{~K}$ were respectively

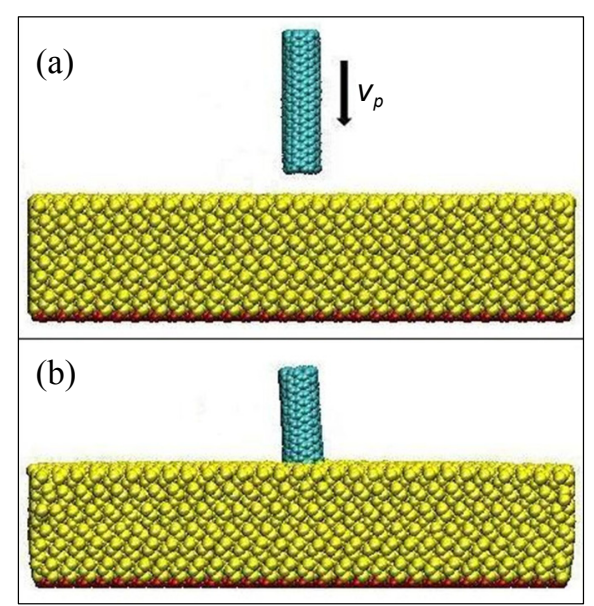

Figure 1. (a) Snapshot of CNT impacting onto a $\mathrm{Si}$ (110) surface with a projectile speed of $v_{p}$ along its axis. The arrow denotes the moving direction of CNT. In this case, $\mathrm{T}_{\mathrm{CNT}}=100 \mathrm{~K}, \mathrm{~T}_{\mathrm{Si}}=300 \mathrm{~K}$, and $v_{p}=5$ $\mathrm{km} / \mathrm{s}$. (b) Snapshot of CNT penetrated into the surface after collision (at 22 ps after impact). 
considered. After being equilibrated to a desired temperature $\mathrm{T}_{\mathrm{CNT}}$, a projectile velocity $v_{p}$ was added to CNT for its collision with the surface. The collision was simulated using a constant energy (NVE) MD method. ${ }^{21}$ Figure $1 \mathrm{~b}$ shows a CNT deposited on the surface after collision (at $22 \mathrm{ps).}$

We used the Tersoff potential ${ }^{22}$ to model the interaction between the CNT and surface. The interatomic potential between atoms $i$ and $j, V_{i j}$, is given by

$$
V_{i j}=f_{C}\left(r_{i j}\right)\left[V_{R}\left(r_{i j}\right)+b_{i j} V_{A}\left(r_{i j}\right)\right],
$$

where $r_{i j}$ represents the interatomic distance. The repulsive and attractive energies, $V_{R}\left(r_{i j}\right)$ and $V_{A}\left(r_{i j}\right)$, respectively, are represented as,

$$
\begin{aligned}
& V_{R}\left(r_{i j}\right)=A_{i j} \exp \left(-\lambda r_{i j}\right), \\
& V_{A}\left(r_{i j}\right)=-B_{i j} \exp \left(-\mu r_{i j}\right) .
\end{aligned}
$$

In equation (1), the cutoff function $f_{C}\left(r_{i j}\right)$ limits the interaction as

$$
f_{C}=\left\{\begin{array}{lr}
1 & \left(r_{i j}<R_{i j}\right) \\
\frac{1}{2}+\frac{1}{2} \cos \left[\frac{\pi\left(r_{i j}-R_{i j}\right)}{S_{i j}-R_{i j}}\right] & \left(R_{i j}<r_{i j}<S_{i j}\right) . \\
0 & \left(r_{i j}>S_{i j}\right)
\end{array}\right.
$$

$b_{i j}$ is the bond order of interaction and is given by

$$
b_{i j}=\chi_{i j}\left(1+\beta_{i}^{n_{i}} \xi_{i j}^{n_{i}}\right)^{-1 / 2 n_{i}},
$$

with

$$
\xi_{i j}=\sum_{k \neq i, j} f_{C}\left(r_{i k}\right) \omega_{i k} g\left(\theta_{i j k}\right)
$$

$g\left(\theta_{i j k}\right)$ is given by

$$
g\left(\theta_{i j k}\right)=1+\frac{c_{i}^{2}}{d_{i}^{i}}-\frac{\mathrm{c}_{\mathrm{i}}^{2}}{\mathrm{~d}_{\mathrm{i}}^{2}+\left(h_{i}-\cos \theta_{i j k}\right)^{2}} .
$$

All the symbols not explained above are parameters which can be calculated by using the following combination rules:

$$
\begin{aligned}
& \lambda_{i j}=\left(\lambda_{i}+\lambda_{j}\right) / 2, \mu_{\mathrm{ij}}=\left(\mu_{\mathrm{i}}+\mu_{\mathrm{j}}\right) / 2 . \\
& A_{i j}=\left(A_{\mathrm{i}} A_{\mathrm{j}}\right)^{1 / 2}, B_{\mathrm{ij}}=\left(B_{i} B_{j}\right)^{1 / 2} . \\
& R_{i j}=\left(R_{\mathrm{i}} R_{\mathrm{j}}\right)^{1 / 2}, S_{\mathrm{ij}}=\left(S_{i} S_{j}\right)^{1 / 2} .
\end{aligned}
$$

The parameters, $\lambda_{i}, \mu_{i}, A_{i}, B_{i}, R_{i}$, and $S_{i}$, for $\mathrm{Si}$ and $\mathrm{C}$ are listed in Reference 22.

The MD trajectory was propagated using the velocity Verlet $\operatorname{algorithm~}^{21}$ with a time step of 0.2 or $0.4 \mathrm{fs}$. The time of impact was defined as the time at which the CNT approaches within $0.178 \mathrm{~nm}$ of the surface $(0.178 \mathrm{~nm}$ is the C-Si distance in a silicon carbide nanotube ${ }^{23}$ ). The MD simulation typically ran for 22 ps after impact. The above MD methods were implemented using the DL POLY package. ${ }^{24}$

The energy of CNT was analyzed as follows. At each time of simulation, the position and velocity of the center of mass were calculated. The internal position and velocity of the $i$ th $C$ atom, $\vec{r}_{i}$ and $\vec{v}_{i}$, were obtained by subtracting these values from the position and velocity of each $\mathrm{C}$ atom, respectively. The internal kinetic energy $K_{\text {int }}$ is given by the sum over each atom as

$$
K_{\mathrm{int}}=(m / 2) \sum_{i} \vec{v}_{i}^{2}
$$

The angular momentum $\vec{L}$ and the moment of the inertia tensor $I$ were also calculated from $\vec{r}_{i} \mathrm{~s}$ and $\vec{v}_{i} \mathrm{~s}$. The rotational kinetic energy $K_{r o t}$ and vibrational kinetic energy $K_{v i b}$ were calculated as

$$
\begin{aligned}
& K_{r o t}=(1 / 2)\left(I^{-1} \vec{L}\right) \cdot \vec{L}, \\
& K_{v i b}=K_{\text {int }}-K_{r o t} .
\end{aligned}
$$

The vibrational temperature $T_{v i b}$ is given by

$$
T_{v i b}=\left(2 K_{v i b}\right) /\left[(3 \times 384-6) k_{B}\right] \text {, }
$$

where $k_{B}$ is the Boltzmann constant.

We quantified the deformation of the CNT by calculating the root-mean-squared displacement (RMSD). To do so, the displacement of each $\mathrm{C}$ atom from its initial value was calculated. The average of the displacement squared was taken by summing over all the $\mathrm{C}$ atoms. We used the numerical method $\mathrm{Kabsch}^{25}$ implemented in the Visual Molecular Dynamics package. ${ }^{26}$

\section{Results and Discussion}

We inspect the snapshots for the deposition of CNT on Si (110) surface which were equilibrated at temperatures of $\mathrm{T}_{\mathrm{CNT}}$ and $\mathrm{T}_{\mathrm{Si}}$, respectively, before collision. Figure 1a shows the CNT impacting on the Si surface at a projectile speed of $v_{p}=5$ $\mathrm{km} / \mathrm{s}$. In this case, $\mathrm{T}_{\mathrm{CNT}}$ and $\mathrm{T}_{\mathrm{Si}}$ are 100 and $300 \mathrm{~K}$, respectively. Figure $1 \mathrm{~b}$ shows the CNT penetrated into the Si surface and vertically aligned. Figures $2 \mathrm{a}-\mathrm{d}$ shows the deformation of CNT for different combinations of $\mathrm{T}_{\mathrm{CNT}}$ and $\mathrm{T}_{\mathrm{Si}}$. In all cases, the $\mathrm{CNT}$ penetrated into the Si surface and were vertically aligned on the surface. The deformation was localized around the bottom of the CNT. In the case of $\mathrm{T}_{\mathrm{CNT}}=100 \mathrm{~K}$ and $\mathrm{T}_{\mathrm{Si}}=100 \mathrm{~K}$, the bottom of CNT is slightly squeezed without much deformation (Figure 2a). As $\mathrm{T}_{\mathrm{Si}}$ increases to $300 \mathrm{~K}$, the bottom atoms of CNT are significantly displaced from their original positions before collision (Figure 2c). On the other hand, increasing the temperature of CNT from 100 to $300 \mathrm{~K}$ does not much affect the deformation: the structure of CNT for $\mathrm{T}_{\mathrm{CNT}}=300 \mathrm{~K}$ and $\mathrm{T}_{\mathrm{Si}}=$ $100 \mathrm{~K}$ (Figure $2 \mathrm{~b})$ is similar to Figure $2 \mathrm{a}\left(\mathrm{T}_{\mathrm{CNT}}=100 \mathrm{~K}\right.$ and 


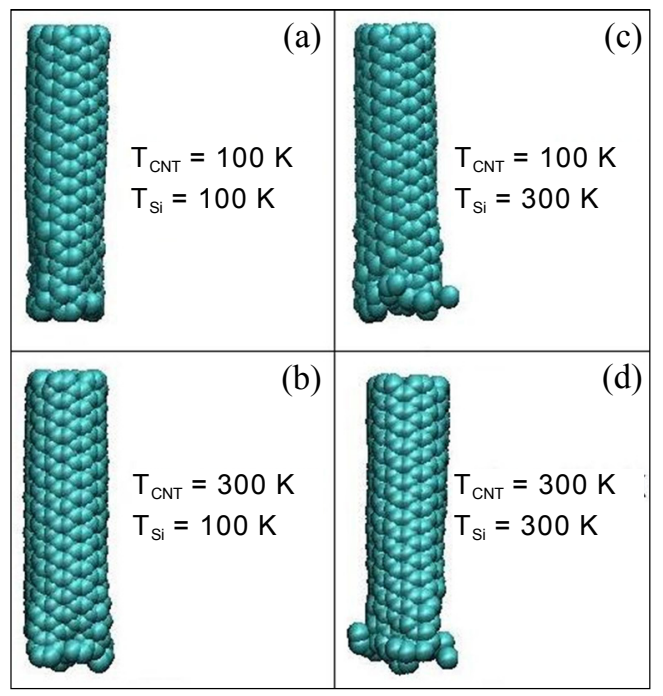

Figure 2. Snapshots of CNTs deformed after collision with $\mathrm{Si}$ (110) surface. Shown are four different combinations of the CNT $\left(\mathrm{T}_{\mathrm{CNT}}\right)$ and surface $\left(\mathrm{T}_{\mathrm{Si}}\right.$ ) temperatures. (a) $\mathrm{T}_{\mathrm{CNT}}=100 \mathrm{~K}$ and $\mathrm{T}_{\mathrm{Si}}=100 \mathrm{~K}$ (b) $\mathrm{T}_{\mathrm{CNT}}=300 \mathrm{~K}$ and $\mathrm{T}_{\mathrm{Si}}=100 \mathrm{~K}$ (c) $\mathrm{T}_{\mathrm{CNT}}=100 \mathrm{~K}$ and $\mathrm{T}_{\mathrm{Si}}=300 \mathrm{~K}$ (d) $\mathrm{T}_{\mathrm{CNT}}=300 \mathrm{~K}$ and $\mathrm{T}_{\mathrm{Si}}=300 \mathrm{~K}$.

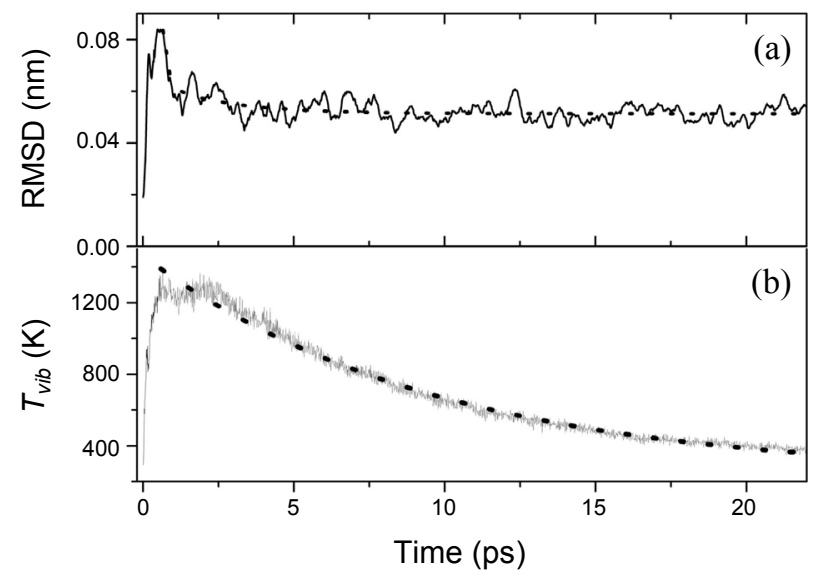

Figure 3. (a) Dynamic deformation of the CNT colliding with the Si (110) surface. For $\mathrm{T}_{\mathrm{CNT}}=300 \mathrm{~K}$ and $\mathrm{T}_{\mathrm{Si}}=150 \mathrm{~K}$, the RMSD was plotted as a function of time elapsed after impact. The RMSD after its maximum is fitted to an exponential function of time (dotted line). (b) Vibrational temperature, $T_{v i b}, v s$. time. $T_{v i b}$ after its maximum is fitted to an exponential function of time (dotted line).

$\left.\mathrm{T}_{\mathrm{Si}}=100 \mathrm{~K}\right)$. The largest deformation of CNT occurs when both the CNT and surface temperatures are high $\left(\mathrm{T}_{\mathrm{CNT}}=300 \mathrm{~K}\right.$ and $\mathrm{T}_{\mathrm{Si}}=300 \mathrm{~K}$, Figure $2 \mathrm{~d}$ ). We did not observe any detachment of the atoms from CNT.

In Figure 3, we plot the RMSD and $T_{v i b} v s$. time for the case of $\mathrm{T}_{\mathrm{CNT}}=300 \mathrm{~K}$ and $\mathrm{T}_{\mathrm{Si}}=150 \mathrm{~K}$. After impact (time zero in the figure), both the RMSD and $T_{v i}$ rapidly increase and reach maxima (about $0.085 \mathrm{~nm}$ and $1381 \mathrm{~K}$, respectively). These initial increases in RMSD and $T_{v i b}$ represent the deformation of CNT and its excitation in vibrational energy, respectively. ${ }^{20} \mathrm{The}$ maxima in RMSD and $T_{v i b}$ are followed by relaxation, in which the RMSD or $T_{v i b}$ decreases and levels off to a finite value. This relaxation was modeled by fitting the RMSD and $T_{v i b}$ curves to (a)
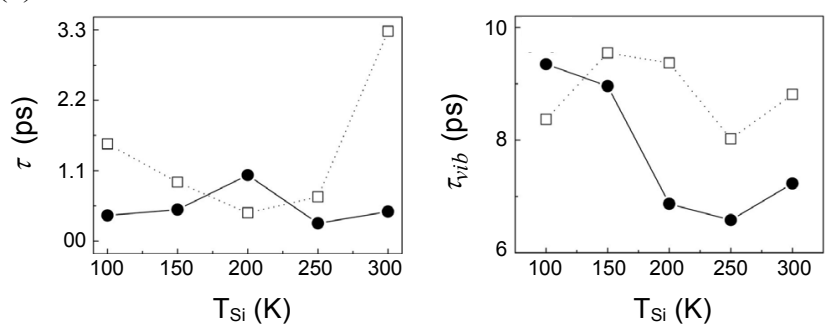

(b)

(d)
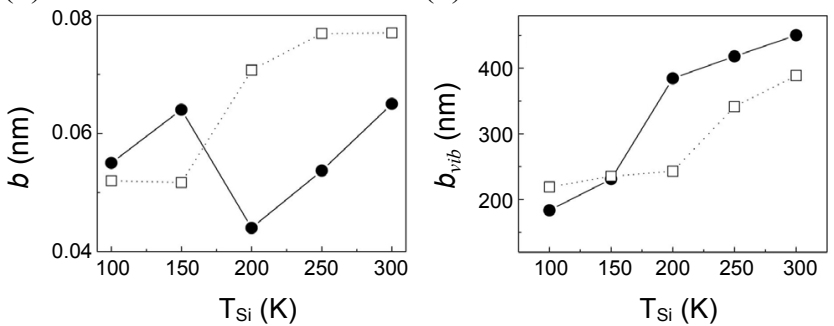

Figure 4. Surface temperature dependence of the deformation and vibrational temperature. In all of the panels, the open squares and filled circles refer to the data obtained for $\mathrm{T}_{\mathrm{CNT}}=300 \mathrm{~K}$ and $\mathrm{T}_{\mathrm{CNT}}$ $=100 \mathrm{~K}$, respectively. Lines are for visual guide. The surface temperature $\mathrm{T}_{\mathrm{Si}}$ is varied as $100 \mathrm{~K}, 150 \mathrm{~K}, 200 \mathrm{~K}, 250 \mathrm{~K}$ and $300 \mathrm{~K}$. (a) The structural relaxation time $\tau v s$. $\mathrm{T}_{\mathrm{Si}}$ (b) The asymptotic RMSD $b v s . T_{\mathrm{Si}}$. (c) The vibrational energy relaxation time $\tau_{v i b} v s . \mathrm{T}_{\mathrm{Si}}(\mathrm{d}) \mathrm{The}$ long time vibrational temperature $b_{v i b} v s$. $\mathrm{T}_{\mathrm{Si}}$.

$a \exp (-t / \tau)+b$ and $a_{v i b} \exp \left(-t / \tau_{v i b}\right)+b_{v i b}$, respectively (shown as dotted lines in Figures $3 \mathrm{a}$ and $\mathrm{b}$ ). The fitting parameters $\tau$ and $\tau_{v i b}$, respectively, are called the structural and energy relaxation times of CNT. $b_{v i b}(529 \mathrm{~K}$ in this case $)$ and $b(0.14 \mathrm{~nm}$ in this case) are the long-time $T_{v i b}$ and RMSD which represents the permanent rise in the vibrational temperature and the permanent deformation, respectively.

We calculated the time dependent RMSD and $T_{v i b}$ for various combinations of $\mathrm{T}_{\mathrm{CNT}}(100$ and $300 \mathrm{~K})$ and $\mathrm{T}_{\mathrm{Si}}(100,200,250$, and $300 \mathrm{~K}$ ). The RMSD and $T_{v i b}$ curves for each combination were fitted to exponential functions as in Figure 3. Such fittings gave us the relaxation times, $\tau$ and $\tau_{v i b}$, and the long time values, $b$ and $b_{v i b}$ which are shown in Figure 4.

Let us examine the temperature dependence of the deformation dynamics of CNT. Figure 4a plots the structural relaxation time $\tau v s$. $\mathrm{T}_{\mathrm{Si}}$ for $\mathrm{T}_{\mathrm{CNTS}}$ of $100 \mathrm{~K}$ (filled circles) and $300 \mathrm{~K}$ (open squares). $\tau$ ranges from 0.4 to $3.3 \mathrm{ps}$ for $\mathrm{T}_{\mathrm{CNT}}=300 \mathrm{~K}$ and from 0.4 to $1.0 \mathrm{ps}$ for $\mathrm{T}_{\mathrm{CNT}}=100 \mathrm{~K}$. Overall, $\tau \mathrm{s}$ for $\mathrm{T}_{\mathrm{CNT}}=300 \mathrm{~K}$ are larger than those for $\mathrm{T}_{\mathrm{CNT}}=100 \mathrm{~K}$, indicating the structural relaxation of the low temperature CNT is faster. The dependence of $\tau$ on $\mathrm{T}_{\mathrm{Si}}$ is opposite for the high and low temperature CNTs: $\tau$ of the high temperature CNT is minimal at $\mathrm{T}_{\mathrm{Si}}=200 \mathrm{~K}$, while $\tau$ for the low temperature CNT is maximal at the same $\mathrm{T}_{\mathrm{Si}}$. The permanent deformation in the CNT was quantified as $b$ which is plotted as a function of $\mathrm{T}_{\mathrm{Si}}$ in Figure $4 \mathrm{~b} . b$ for the high temperature CNT generally increases with raising $T_{\mathrm{Si}}$ while $b$ is not a monotonic function of $\mathrm{T}_{\mathrm{Si}}$ for the low temperature $\mathrm{CNT}$. On the whole, $b$ is greater for the high temperature CNT, but this difference is small.

Moving on to the dynamics of vibrational temperature, $\tau_{v i b}$ 
was plotted as a function of $\mathrm{T}_{\mathrm{Si}}$ in Figure $4 \mathrm{c} . \tau_{v i b}$ ranged from 6.6 to $9.3 \mathrm{ps}$ for $\mathrm{T}_{\mathrm{CNT}}=100 \mathrm{~K}$ and from 8.0 to $9.6 \mathrm{ps}$ for $\mathrm{T}_{\mathrm{CNT}}=$ $300 \mathrm{~K}$. On the whole, $\tau_{v i b}$ is greater for the high temperature CNT as found for $\tau$ above, implying that the vibrational relaxation is faster for the low temperature CNT. No clear trend is found for its dependence on $\mathrm{T}_{\mathrm{Si}}$, although it seems decreasing with the rise in $\mathrm{T}_{\mathrm{Si}}$ for the low temperature $\mathrm{CNT}$. Figure $4 \mathrm{~d}$ plots $b_{v i b}$, as a function of $\mathrm{T}_{\mathrm{Si}} . b_{v i b}$ increases with the rise in $\mathrm{T}_{\mathrm{Si}}$, regardless of $\mathrm{T}_{\mathrm{CNT}}$. $b_{v i b}$ increases from 183 to $450 \mathrm{~K}$ in the case of $\mathrm{T}_{\mathrm{CNT}}=100 \mathrm{~K}$ and from 219 to $389 \mathrm{~K}$ for $\mathrm{T}_{\mathrm{CNT}}=300 \mathrm{~K}$. Interestingly, the low temperature $\mathrm{CNT}\left(\mathrm{T}_{\mathrm{CNT}}=100 \mathrm{~K}\right)$ has $b_{v i b}$ s higher than those of the high temperature CNT. Presumably, heat is transferred from the surface to the low temperature $\mathrm{CNT}$, giving an increased vibrational temperature. On the other hand, a high temperature CNT dissipates its energy to the surface and therefore has a lower value of $b_{v i b}$.

\section{Conclusion}

By using MD simulation, we studied the temperature dependent deposition of CNTs on a silicon surface via collision. By focusing on the collision geometry where the CNT impacts onto the surface along its long axis, we investigated the effects of the CNT temperature and the surface temperature. The CNT was vertically aligned on the surface and penetrated up to a depth of $1.2 \mathrm{~nm}$ at a projectile speed $v_{p}=5 \mathrm{~km} / \mathrm{s}$. Due to its high mechanical resilience, the CNT shows only a small deformation localized at its bottom contacting the surface. The structural and energy relaxation of the low temperature CNT was more efficient (faster) than that of the high temperature CNT. The vibrational temperature of CNT increased with the rise in the surface temperature, regardless of its temperature. A low temperature CNT ended up with a higher vibrational temperature after collision than a high temperature CNT. This is probably due to the possible heat transfer from the surface to the CNT via collision.

By averaging over the entire atoms of CNT in calculating the RMSD and vibrational temperature, we left out the dynamics of intramolecular vibrational energy redistribution (IVR). A study of IVR via a normal or local mode analysis will elucidate the pathways of the energy (heat) transfer from the bottom to top of CNT after impact. We leave this IVR study as an interesting future work.
Acknowledgments. This work was supported for two years by a Pusan National University Research Grant.

\section{References}

1. Ye, H.; Lam, H.; Titchenal, N.; Gogotsi, Y.; Ko, F. Appl. Phys. Lett. 2004, 85, 1775 .

2. Siegel, R. W.; Chang, S. K.; Ash, B. J.; Stone, J.; Ajayan, P. M.; Doremus, R. W.; Schadler, L. S. Scripta Materialia 2001, 44, 2061.

3. Thostenson, E. T.; Ren, Z.; Chou, T.-W. Composites Science and Technology 2001, 61, 1899.

4. Cha, S. I.; Kim, K. T.; Lee, K. H.; Mo, C. B.; Hong, S. H. Scripta Materialia 2005, 53, 793.

5. Qi, D.; Hinkley, J.; He, G. Modelling Simul. Mater. Sci. Eng. 2005, 13, 493.

6. Frankland, S. J. V.; Harik, V. M.; Odegard, G. M.; Brenner, D. W.; Gates, T. S. Composites Science and Technology 2003, 63, 1655.

7. Mylvaganam, K.; Zhang, L. C. Appl. Phys. Lett. 2006, 89, 123127.

8. Laha, T.; Agarwal, A. Materials Science and Engineering A 2008, 480, 323.

9. Laha, T.; Agarwal, A.; Mckechnie, T.; Seal, S. Materials Science and Engineering $A$ 2004, 381, 249.

10. Li, C.-J.; Li, W.-Y.; Wang, Y.-Y. Surface and Coatings Technology 2005, 198, 469.

11. Balani, K.; Laha, T.; Agarwal, A.; Karthikeyan, J.; Munroe, N. Surface and Coatings Technology 2005, 195, 272.

12. Bakshi, S. R.; Singh, V.; McCartney, D. G.; Seal, S.; Agarwal, A. Scripta Materialia 2008, 59, 499.

13. Bakshi, S. R.; Singh, V.; Balani, K.; McCartney, D. G.; Seal, S.; Agarwal, A. Surface and Coatings Technology 2008, 202, 5162.

14. Lopez, M. J.; Cabria, I.; March, N. H.; Alonso, J. A. Carbon 2005, 43, 1371.

15. Liew, K. M.; Wong, C. H.; He, X. Q.; Tan, M. J. Phys. Rev. B 2005, 71,075424

16. Che, J.; Cagin, T.; Goddard, W. A. Nanotechnology 2000, 11, 65.

17. Bi, K.; Chen, Y.; Yang, J.; Wang, Y.; Chen, M. Physics Letters A 2006, 350, 150.

18. Cheng, M.; Lu, Y. IEEE Transactions on Magnetics 2006, $42,891$.

19. Yin, H.; Luo, C. Modelling Simulation Mater. Sci. Eng. 2005, 13, 1403.

20. Saha, L. C.; Mian, S. A.; Kim, H.; Jang, J. J. Phys. Chem. C 2009, $113,16668$.

21. Allen, M. P.; Tildesley, D. J. Computer Simulation of Liquids; Clarendon Press: Oxford, U.K, 1987.

22. Tersoff, J. Phys. Rev. B 1989, 39, 5566.

23. Zhao, J.-X.; Ding, Y.-H. J. Chem. Theory Comput. 2009, 5, 1099.

24. Smith, W.; Yong, C. W.; Rodger, P. M. Mol. Simul. 2002, $28,385$.

25. Kabsch, W. Acta Crystallogr. Sect. A 1978, 34, 827.

26. Humphrey, W.; Dalke, A.; Schulten, K. J. Mol. Graphics 1996, 14,33 . 